\title{
Correction to: Platelets, Haemostasis and Inflammation
}

Andreas Zirlik, Christoph Bode, and Meinrad Gawaz

\section{Correction to:}

\section{A. Zirlik et al. (eds.), Platelets, Haemostasis and Inflammation, Cardiac and Vascular Biology 5, https://doi.org/10.1007/978-3-319-66224-4}

The original version of this volume was revised as it was originally published unnumbered. The revised version now has been numbered; numbering is done following the order of appearance in the book series Cardiac and Vascular Biology. 